\title{
Traumatic pancreatitis with peripheral fat necrosis
}

\author{
K. M. COCHRAN \\ M.B., M.R.C.P., D.R.C.O.G. \\ J. R. MASON
M.B., F.R.C.S. \\ J. S. H. DAVIDSON \\ M.B., F.F.R. \\ Divisions of Medicine, Surgery and Radiology, Glasgow Victoria Infirmary
}

\begin{abstract}
Summary
Direct damage to the pancreas occurs in about $5 \%$ of closed abdominal injuries, many of which arise from major accidents. The fixed retro-peritoneal site of the pancreas makes it particularly vulnerable to trauma by the steering column in cars not being constructed to modern safety requirements. As these injuries become more common, and as the number of survivors of such trauma increases, the longer term complications of trauma to the pancreas will become more common. A case is described demonstrating some of these complications arising from peripheral fat necrosis.
\end{abstract}

\section{Case report}

A 54-year-old labourer was admitted to the Victoria Infirmary following a steering wheel injury to the epigastrium. Initially, he was found to have guarding in the upper abdomen. His blood pressure was $120 / 180$ and his pulse rate $70 / \mathrm{min}$. A plain radiograph of chest and abdomen showed no abnormality. Within an hour of admission there were obvious signs of peritoneal irritation, and the patient complained of shoulder tip pain. His pulse rate had risen to $100 / \mathrm{min}$ and his blood pressure has fallen to $100 / 60 \mathrm{mmHg}$. With a presumptive diagnosis of a rupture of the spleen the patient underwent laparotomy.

\section{Operative findings}

The abdomen contained $600 \mathrm{ml}$ of blood. The liver, spleen and mesentery were all normal. A collection of blood could be seen through the lesser omentum which was opened to give access to the lesser sac. The intact splenic artery was immediately visible together with three small pancreatic branches

Correspondence: Dr K. M. Cochran, Senior Registrar, Ward 8C, Gartnaval General Hospital, 1053 Great Western Road, Glasgow G12 0YN. which had been avulsed from the tail of the pancreas where there was some contusion, but no obvious rupture. These bleeding points were ligated and haemorrhage controlled. No other abnormality was found in the rest of the abdomen. A large drain was placed down to the pancreas and the wound closed in layers. The recovery of the patient was remarkably uneventful apart from a slight pyrexia which settled spontaneously. He was discharged from hospital 12 days later.

The patient was re-admitted to a medical ward 18 days later complaining of rigors; of swelling and pain of both feet, ankles and dorsum of the left hand; and of multiple black non-tender subcutaneous nodules. Physical examination revealed an ill man with temperature of $38.4^{\circ} \mathrm{C}$ and pulse $120 / \mathrm{min}$. Abdominal examination revealed no abnormalities. The striking features were erythema and painful swelling of the left hand (Fig. 1) and both ankles; and multiple black non-tender nodules on lower legs, thigh and extensor aspect of arms (Fig. 2).

\section{Investigation}

The following investigations were performed: haemoglobin $13.2 \mathrm{~g} / 100 \mathrm{ml}$, white blood cell count 18,200 cells $/ \mathrm{mm}^{3}$ ( $85 \%$ neutrophils), ESR $111 \mathrm{~mm}$ in the first hour, blood urea $33 \mathrm{mg} / 100 \mathrm{ml}$, chloride $98 \mathrm{mEq} / \mathrm{l}$, sodium $137 \mathrm{mEq} / \mathrm{l}$, potassium $3.2 \mathrm{mEq} / \mathrm{l}$, total plasma protein $6 \mathrm{~g} / 100 \mathrm{ml}$, albumin $1.54 \mathrm{~g} /$ $100 \mathrm{ml}$. Electrophoresis showed hypoalbuminaemia with increase in $\alpha 1$, and $\alpha 2$ globulins. Serum amylase was 624 van Loon u/ml (normal <35). Serum lipase was 5 Cherry Crandall $\mathrm{u} / \mathrm{ml}$ (normal $<1$ ). Blood cultures done on six occasions failed to grow any organism. Serum uric acid was $1.8 \mathrm{mg} / 100 \mathrm{ml}$ and anti-streptolysin-O-titre was 100 Todd $u$. The WR, GCFT, LRA, and ANF were all negative. Electrocardiography and radiography of the chest, hands, 


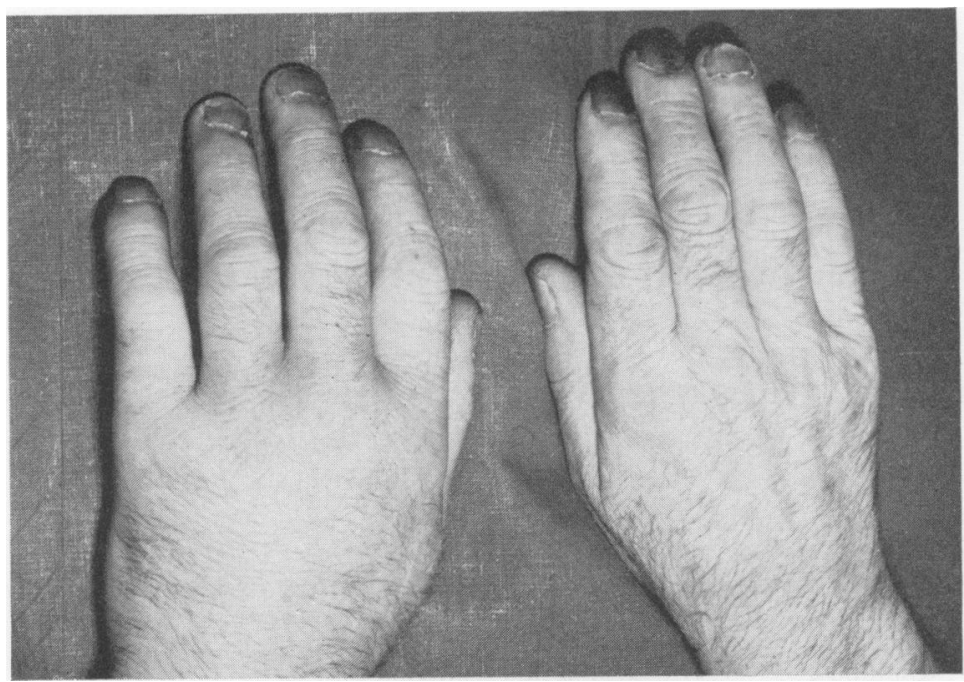

Fig. 1. Appearances of left hand during acute stages of illness showing marked swelling and erythema of dorsum.

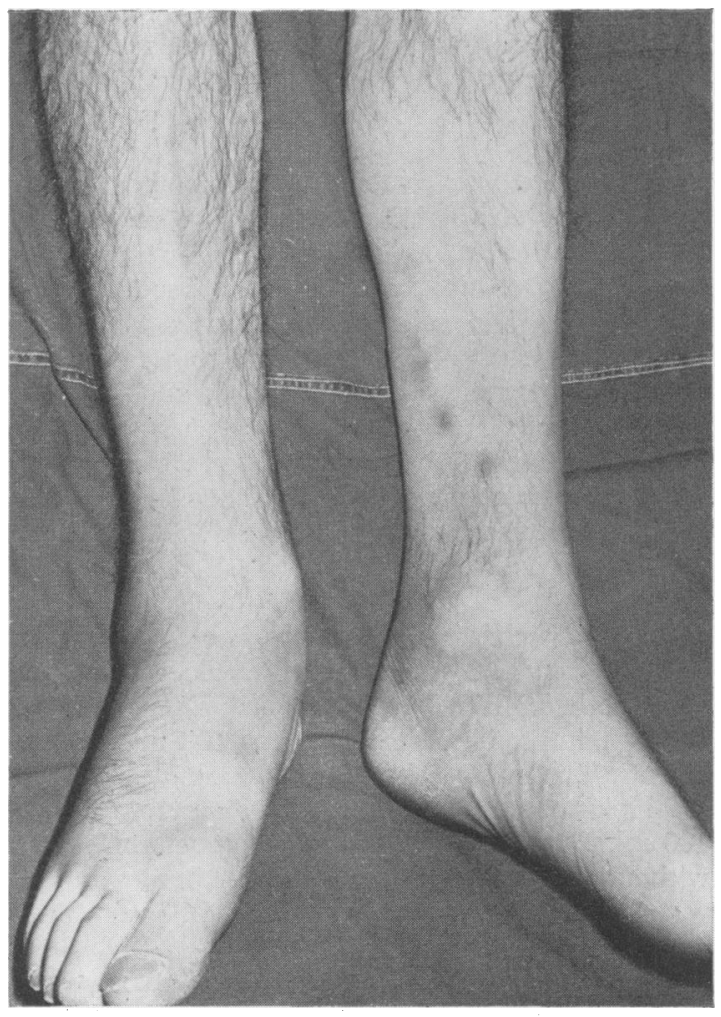

FIG. 2. Appearances of both ankles showing oedema, erythema, and subcutaneous fat necrosis above left medial malleolus. and ankles showed no abnormality. Biopsy of a subcutaneous lesion from lower leg showed acute fat necrosis, the fat in the area being completely replaced by a homogeneous granular or fibrillary basophilic material and a few fatty acid crystals and soaps.

\section{Course in hospital}

For 8 days after admission the patient had an intermittent pyrexia which did not settle in spite of intravenous cephaloridine and later steroid therapy. A barium meal examination at this time failed to reveal any evidence of a pancreatic cyst and a plain radiograph of the chest and diaphragmatic screening failed to show a subphrenic abscess. In spite of this it was felt that there was a source of sepsis inside the abdomen responsible for his high swinging temperature and metastatic lesions, and the patient therefore had an exploratory laparotomy.

The patient returned to theatre 42 days after his first operation. The omentum was adherent to the old incision. The peritoneal cavity was free of general adhesions and there was no subphrenic abscess present. The left lobe of the liver was adherent to the lesser omentum and in the lesser sac there were about $10 \mathrm{ml}$ of turbid fluid surrounded by obvious fat necrosis. The tail of the pancreas was indurated. A large drain was again placed into the lesser sac and the abdomen closed. Following surgery there was an immediate fall in lipase and amylase levels, which were normal within $48 \mathrm{hr}$. The abdominal wound healed without fistula formation, and the patient's temperature returned to normal. The arthropathy 
remained unchanged. The patient became confused and showed personality changes which lasted for several weeks. Now 2 months after re-admission $\mathrm{X}$-ray changes were apparent at both ankles. These consisted of soft tissue swelling with small areas of calcification within the swollen areas. The joint space at the left ankle was narrowed. Three months after re-admission, having regained mental function, he was allowed home. Shortly before discharge a skeletal survey was performed. Numerous small osteolytic defects were demonstrated in the phalanges and metacarpals of hands and feet (Fig. 3). Similar lesions were present in the distal radii and ulnae. No abnormality was seen elsewhere in the skeleton.

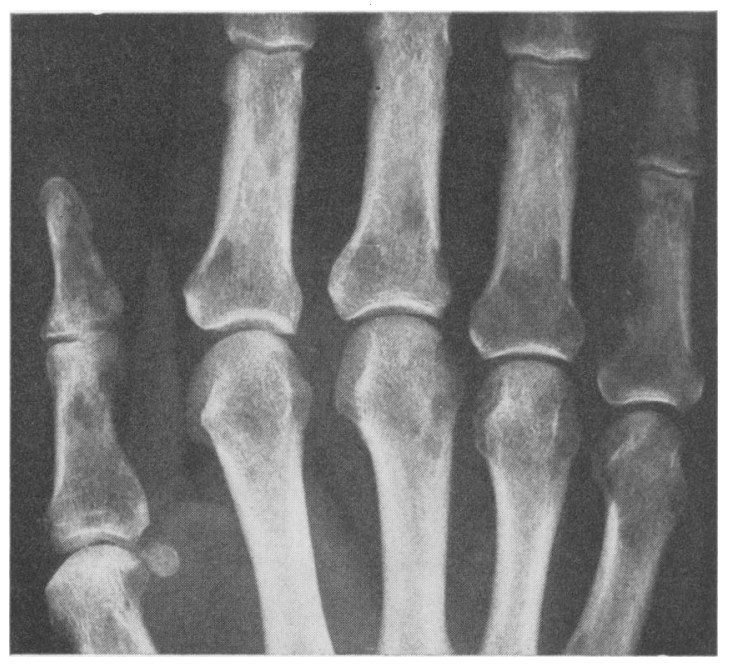

FIG. 3. Radiograph of right hand showing osteolytic lesions of small bones, typical of fat necrosis.

\section{Subsequent progress}

Follow-up has shown him to have had no abdominal symptoms and his blood chemistry has remained normal. The X-ray changes in the hand have regressed, but residual arthritic changes remain at both ankles. Radiographs of lower femora 18 months after the accident did not show any intramedullary calcification.

\section{Discussion}

Chiari (1883) first recognized the relationship between peripheral skin lesions and pancreatic disease. Mullin et al. (1968) reviewed the then thirty-six reported cases of pancreatic disease with multiple sites of fat necrosis. Few had involvement as extensive as the case recorded above, and certainly such an extensive process is usually associated with a poor prognosis. Fat necrosis has been described
TABle 1. Reported clinical and autopsy sites of fat necrosis

\begin{tabular}{l} 
Pancreas \\
Peripancreatic \\
Omental \\
Retroperitoneal \\
Perinephric \\
Mediastinal \\
Subpleural \\
Subepicardial \\
Subcutaneous \\
Bone marrow \\
Periarticular \\
Central nervous system \\
(Perivascular demyelinization) \\
\hline
\end{tabular}

clinically by Lucas and Owen (1962), Swerdlow et al. (1960), Kushner and Szanto (1958) and Mullin et al. (1968); and at autopsy by Scarpelli (1956), Roberts, Baggenstoss and Comfort (1950) and Vogel (1951), in sites listed in Table 1.

This patient demonstrated intra-abdominal, subcutaneous and periarticular fat necrosis. In addition, we think he exhibited perivascular demyelinization as manifest by his bizarre mental state during the acute illness. Originally, alcohol withdrawal or hepatic dysfunction was thought to be the cause of his symptoms but in view of the other findings this now seems unlikely. Mullin et al. (1968) found a high alcohol intake in $40 \%$ of cases, and this patient could fit into such a group.

The subcutaneous lesions closely resemble erythema nodosum. Other conditions to be excluded include allergic vasculitis, acute thrombophlebitis, cellulitis and local trauma. Distinguishing features from erythema nodosum include the site of involvement and the breakdown of the lesions which discharge a creamy material high in pancreatic enzyme activity.

The abnormal findings on X-ray examination include soft tissue swelling, soft tissue calcification, and narrowing of joint spaces at the ankles. The other abnormal radiographic feature was the small osteolytic lesions in hands and feet. All these findings can be explained by fat necrosis. The osteolytic lesions are thought to have been caused by medullary fat necrosis. The location of the defects in the small bones is explained by Immelman et al. (1964) as being due to the relatively small volume of medullary cavity in small bones allowing a destruction of adjacent cortical bone.

The appearance and site of the osteolytic defects are not in keeping with rheumatoid arthritis or gout, nor is the fact that the lesions have almost completely regressed 18 month's after the accident. The calcification seen at times in the intramedullary cavity of lower femora in cases of chronic pancreatitis has not appeared in this patient. 
The likeliest explanation for these fatty lesions is that they are produced by pancreatic enzymes which have entered the circulation either directly or via lymphatics. The latter is thought to be the more important route. Abdominal peritoneal lymphatic drainage and transdiaphragmatic lymphatic absorption of abdominal fluid are probably prime factors in the ingress of pancreatic enzymes to the thoracic duct and hence into general circulation (Scarpelli, 1956; Perry, 1947). Circulatory trypsin may break down vessel wall integrity and allow lipase to hydrolyse neutral fat in fat cells to glycerol and fatty acid. It has also been suggested (Hodson-Walker and Woods, 1970) that the lesions are secondary to vascular spasm or necrosis, which leads to local areas of fat necrosis. The primary insult on the vessel being from pancreatic enzymes or due to intravascular thrombosis sometimes seen in acute pancreatitis.

A further but unlikely factor is that the lesions are caused by emboli of necrotic pancreatic tissue (Blauvelt, 1946).

Peripheral fat necrosis has been described in acute pancreatitis and as a marker of pancreatic neoplasm. This case and the others described should serve as a reminder that pancreatic disease may present with bizarre and obscure symptoms and signs.

\section{Acknowledgments}

We would like to thank Dr M. J. Riddell and Mr G. Smillie for permission to publish and Dr A. Glen for invaluable help with biochemical data.

\section{References}

Blauvelt, H. (1946) Case of acute pancreatitis with subcutaneous fat necrosis. British Journal of Surgery, 34, 207.

ChIari, H. (1883) Quoted in Mullin, G.T. and others (1968). Annals of Internal Medicine, 68, 75.

Hodson-Walker, N.J. \& Woods, J.M. (1970) Acute pancreatitis with peripheral fat necrosis. Canadian Medical Association Journal, 103, 382.

ImmelmaN, E.J., BANKS, S., KRIGe, H. \& MARKS, I.M. (1964) Roentgenologic and clinical features of intramedullary fat necrosis in bones in acute and chronic pancreatitis. American Journal of Medicine, 36, 96.

Kushner, S.D. \& Szanto, P.B. (1958) Fulminant polyarthritis, fever, and cutaneous nodules in alcoholic patients. Journal of the American Medical Association, 167, 1625.

LUCAS, P.F. \& OWEN, T.K. (1962) Subcutaneous fat necrosis, 'polyarthritis' and pancreatic disease. Gut, 3, 146.

Mullin, G.T., Caperton, M.E., Crespin, S.R. \& Williams, R.C. (1968) Arthritis and skin lesions resembling erythema nodosum in pancreatic disease. Annals of Internal Medicine, 68, 75.

Perry, T.T. (1947) Role of lymphatic vessels in transmission of lipase in disseminated pancreatic fat necrosis. Archives of Pathology, 43, 456.

Roberts, J.M., BAggenstoss, A.H. \& Comfort, M.W. (1950) Acute pancreatic necrosis. American Journal of Clinical Pathology, 20, 742.

SCARPELLI, D.G. (1956) Fat necrosis of bone marrow in acute pancreatitis. American Journal of Pathology, 32, 1077.

Swerdlow, A.B., Berman, M.E., Gibbel, M.I. \& Valaitis, J. (1960) Subcutaneous fat necrosis associated with acute pancreatitis. Journal of American Medical Association, 173, 765.

VOGEL, S.F. (1951) Cerebral demyelination and focal visceral lesions in a case of acute haemorrhagic pancreatitis. Archives of Pathology, 52, 355.

\title{
Periodic migrainous neuralgia associated with an arteriovenous malformation
}

\author{
A. L. ThOMAS \\ M.B., M.R.C.P. \\ Southmead Hospital, Bristol BS10 5NB
}

\begin{abstract}
Summary
A patient with periodic migrainous neuralgia is reported. An arteriovenous malformation was found on the affected side. This association has not previously been recorded.

\section{Introduction}

In 1926 Wilfred Harris wrote of a type of recurrent facial pain and headache which he called periodic migrainous neuralgia. The syndrome was described

Present address: Medical Unit, Welsh National School of Medicine, Royal Infirmary, Cardiff CF2 1SZ.
\end{abstract}

in greater detail by Symonds (1956). Paroxysms of headache occur in bouts lasting several weeks with long intervals of freedom. There is at least one paroxysm in each $24 \mathrm{hr}$ period. The pain is agonizing and is felt mainly in the supra-orbital region, in and behind the eye, and it is strictly unilateral. The patient is woken from his sleep in the early hours of the morning and cannot lie still. It rarely lasts longer than $2 \mathrm{hr}$ and there is complete relief from pain between the paroxysms. The interval between bouts is rarely less than 6 months and may be several years. Balla and Walton (1964) documented twenty-eight 